\title{
Soundness of Inference Rules for New Vague Multivalued Dependencies
}

\author{
Dženan Gušić ${ }^{1, *}$ \\ ${ }^{1}$ University of Sarajevo, Faculty of Sciences and Mathematics, Department of Mathematics, Zmaja od Bosne 33-35, 71000 Sarajevo, \\ Bosnia and Herzegovina
}

\begin{abstract}
In the present paper we give a new definition of vague multivalued dependencies in database relations. The definition is based on application of arbitrary similarity measure on vague values, which is known to be reflexive, symmetric, and max-min transitive. The definition is adapted in order to include the imprecise and precise vague multivalued dependencies. The inference rules for new vague multivalued dependencies are listed, and are shown to be sound.
\end{abstract}

\section{Introduction}

Let $V$ be a vague set in $U$, where $U$ is some universe of discourse. We have,

$$
V=\left\{\left\langle u,\left[t_{V}(u), 1-f_{V}(u)\right]\right\rangle: u \in U\right\},
$$

where $t_{V}, f_{V}: U \rightarrow[0,1]$ are some functions such that $t_{V}(u)+f_{V}(u) \leq 1$ for all $u \in U$.

The interval $\left[t_{V}(u), 1-f_{V}(u)\right] \subseteq[0,1]$ represents a vague value associated to $u \in U$.

Obviously, if $t_{V}(u)=1-f_{V}(u) \in(0,1)$, the vague value $\left[t_{V}(u), 1-f_{V}(u)\right]$ becomes the fuzzy value $t_{V}(u)$. In particular, $\left[t_{V}(u), 1-f_{V}(u)\right]$ becomes the crisp value 1 if $t_{V}(u)=1-f_{V}(u)=1$. Finally, if $t_{V}(u)=1-f_{V}(u)=$ 0 , then we assume that $u$ is not an element of the vague set $V$.

Let $R\left(A_{1}, A_{2}, \ldots, A_{n}\right)$ be a relation scheme on domains $U_{1}, U_{2}, \ldots, U_{n}$, where $A_{i}$ is an attribute on the universe of discourse $U_{i}, i \in\{1,2, \ldots, n\}=I$. Suppose that $V\left(U_{i}\right)$ is the family of all vague sets in $U_{i}, i \in I$. A vague relation instance $r$ on $R\left(A_{1}, A_{2}, \ldots, A_{n}\right)$ is a subset of the cross product

$$
V\left(U_{1}\right) \times V\left(U_{2}\right) \times \ldots \times V\left(U_{n}\right) .
$$

Suppose that

$$
t\left[A_{i}\right]=\left\{\left\langle u_{i}, a_{u_{i}}^{t}\right\rangle: u_{i} \in U_{i}\right\},
$$

for all $i \in I$, and all tuples $t=\left(t\left[A_{1}\right], t\left[A_{2}\right], \ldots, t\left[A_{n}\right]\right) \in r$, where $r$ is some fuzzy relation instance on $R\left(A_{1}, A_{2}, \ldots, A_{n}\right)$. More precisely, suppose that $a_{u_{i}}^{t} \in[0,1]$ for all $u_{i} \in U_{i}, i \in I$, and all $t \in r$, where $a_{u_{i}}^{t}$ is the membership value of the element $u_{i} \in U_{i}$ to the fuzzy set $t\left[A_{i}\right]$. The value $t\left[A_{i}\right]$ of the attribute $A_{i}$ on the tuple $t$ may be represented as

$$
t\left[A_{i}\right]=\left\{\left\langle u_{i},\left[a_{u_{i}}^{t}, a_{u_{i}}^{t}\right]\right\rangle: u_{i} \in U_{i}\right\} .
$$

*e-mail: dzenang@pmf.unsa.ba
Hence, the fuzzy relation instance $r$ on $R\left(A_{1}, A_{2}, \ldots, A_{n}\right)$ may be represented as a vague relation instance on $R\left(A_{1}, A_{2}, \ldots, A_{n}\right)$.

Similarly, if

$$
t\left[A_{i}\right]=\left\{u_{i}^{t}\right\}
$$

for all $i \in I$, and all tuples $t \in r$, where $u_{i}^{t}$ is some element in $U_{i}$, and $r$ is some relation instance on $R\left(A_{1}, A_{2}, \ldots, A_{n}\right)$, then, we may write

$$
\begin{aligned}
t\left[A_{i}\right]= & \left\langle u_{i}^{t},[1,1]\right\rangle \cup \\
& \left\{\left\langle u_{i},[0,0]\right\rangle: u_{i} \in U_{i} \backslash\left\{u_{i}^{t}\right\}\right\} .
\end{aligned}
$$

Therefore, the relation instance $r$ on $R\left(A_{1}, A_{2}, \ldots, A_{n}\right)$ may also be represented as a vague relation instance on $R\left(A_{1}, A_{2}, \ldots, A_{n}\right)$.

The discussion given above, shows that the vague relational concept generalizes in a natural way both, the classical relational concept as well as the fuzzy relational concept.

For the basic relational concepts, we refer to [12] (see also, [8], [1], [20]).

In [10], we applied the similarity measures defined as follows (see also, [14], [5]-[11], [13], [18], [9]).

Let $x=[a, 1-b] \subseteq[0,1]$ and $y=[c, 1-d] \subseteq[0,1]$ be some vague values. It is not required these values to be associated to the elements of the same universe of discourse.

The similarity measure $S E(x, y)$ between the vague values $x$ and $y$ is given by

$$
\begin{aligned}
& S E(x, y) \\
= & \sqrt{\left(1-\frac{|(a-c)-(b-d)|}{2}\right)(1-|(a-c)+(b-d)|) .}
\end{aligned}
$$

It is known that $S E(x, y) \in[0,1], S E(x, y)=S E(y, x)$, $S E(x, y)=1$ if and only if $x=y$, and $S E(x, y)=0$ if and only if $x=[0,0], y=[1,1]$ or $x=[0,1], y=[q, q], 0 \leq q$ $\leq 1$. 
If

$$
\begin{aligned}
& A=\left\{\left\langle u,\left[t_{A}(u), 1-f_{A}(u)\right]\right\rangle: u \in U\right\}, \\
& B=\left\{\left\langle u,\left[t_{B}(u), 1-f_{B}(u)\right]\right\rangle: u \in U\right\}
\end{aligned}
$$

are two vague sets in some universe of discourse $U$, then, the similarity measure $S E(A, B)$ between the vague sets $A$ and $B$ is given by

$$
\begin{aligned}
& S E(A, B) \\
= & \frac{1}{|U|} \sum_{u \in U} S E\left(\left[t_{A}(u), 1-f_{A}(u)\right],\left[t_{B}(u), 1-f_{B}(u)\right]\right) \\
= & \frac{1}{|U|} \sum_{u \in U} \sqrt{1-\frac{\left|\left(t_{A}(u)-t_{B}(u)\right)-\left(f_{A}(u)-f_{B}(u)\right)\right|}{2}} \times \\
& \sqrt{1-\left|\left(t_{V_{A}}(u)-t_{B}(u)\right)+\left(f_{A}(u)-f_{B}(u)\right)\right|},
\end{aligned}
$$

where $|U|$ is the number of the elements in $U$.

It is easily deduced that $S E(A, B) \in[0,1], S E(A, B)=$ $S E(B, A), S E(A, B)=1$ if and only if $A=B$, and $S E(A, B)=0$ if and only if $\left[t_{A}(u), 1-f_{A}(u)\right]=[0,0]$, $\left[t_{B}(u), 1-f_{B}(u)\right]=[1,1]$ for all $u \in U$ or $\left[t_{A}(u), 1-f_{A}(u)\right]=[0,1],\left[t_{B}(u), 1-f_{B}(u)\right]=[q, q]$, for all $u \in U$, where $0 \leq q \leq 1$.

The equality $A=B$ means that $A \subseteq B$ and $B \subseteq A$, where $A$ is contained in $B$, i.e., $A \subseteq B$ holds true, if and only if $t_{A}(u) \leq t_{B}(u)$ and $1-f_{A}(u) \leq 1-f_{B}(u)$ for all $u \in U$.

So, $A=B$ if and only if $t_{A}(u)=t_{B}(u), f_{A}(u)=f_{B}(u)$ for all $u \in U$.

Finally, if $R\left(A_{1}, A_{2}, \ldots, A_{n}\right)$ is a relation scheme on domains $U_{1}, U_{2}, \ldots, U_{n}$, where $A_{i}$ is an attribute on the universe of discourse $U_{i}, i \in I, r$ is a vague relation instance on $R\left(A_{1}, A_{2}, \ldots, A_{n}\right), t_{1}$ and $t_{2}$ are any two tuples in $r$, and $X \subseteq\left\{A_{1}, A_{2}, \ldots, A_{n}\right\}$ is a set of attributes, then, the similarity measure $S E_{X}\left(t_{1}, t_{2}\right)$ between the tuples $t_{1}$ and $t_{2}$ on the attribute set $X$ is given by

$$
S E_{X}\left(t_{1}, t_{2}\right)=\min _{A \in X}\left\{S E\left(t_{1}[A], t_{2}[A]\right)\right\} .
$$

It is also easily deduced that $S E_{X}\left(t_{1}, t_{2}\right) \in[0,1]$, $S E_{X}\left(t_{1}, t_{2}\right)=S E_{X}\left(t_{2}, t_{1}\right), S E_{X}\left(t_{1}, t_{2}\right)=1$ if and only if $t_{1}\left[A_{k}\right]=t_{2}\left[A_{k}\right]$ for all $A_{k} \in X$ if and only if $t_{t_{1}\left[A_{k}\right]}(u)=t_{t_{2}\left[A_{k}\right]}(u), f_{t_{1}\left[A_{k}\right]}(u)=f_{t_{2}\left[A_{k}\right]}(u)$ for all $u \in$ $U_{k}$, and all $A_{k} \in X$, and $S E_{X}\left(t_{1}, t_{2}\right)=0$ if and only if there exists $A_{k} \in X$ such that $\left[t_{t_{1}\left[A_{k}\right]}(u), 1-f_{t_{1}\left[A_{k}\right]}(u)\right]=$ $[0,0],\left[t_{t_{2}\left[A_{k}\right]}(u), 1-f_{t_{2}\left[A_{k}\right]}(u)\right]=[1,1]$ for all $u \in U_{k}$ or $\left[t_{t_{1}\left[A_{k}\right]}(u), 1-f_{t_{1}\left[A_{k}\right]}(u)\right]=[0,1],\left[t_{t_{2}\left[A_{k}\right]}(u), 1-f_{t_{2}\left[A_{k}\right]}(u)\right]$ $=[q, q]$ for all $u \in U_{k}$, where $0 \leq q \leq 1$.

In [10], we have proved the assertions:

1) $S E_{Y}\left(t_{1}, t_{2}\right) \geq S E_{X}\left(t_{1}, t_{2}\right)$ for $t_{1}, t_{2}$ in $r$, if $Y \subseteq X \subseteq$ $\left\{A_{1}, A_{2}, \ldots, A_{n}\right\}$,

2) $S E_{X}\left(t_{1}, t_{2}\right) \geq \theta$ if $S E\left(t_{1}[A], t_{2}[A]\right) \geq \theta$ for all $A \in$ $X$,

3) $S E_{X}\left(t_{1}, t_{2}\right) \geq \theta$ and $S E_{X}\left(t_{2}, t_{3}\right) \geq \theta$ do not necessarily imply that $S E_{X}\left(t_{1}, t_{3}\right) \geq \theta$, where $t_{1}, t_{2}$ and $t_{3}$ are some mutually distinct tuples in $r$.

In this paper we introduce the similarity measures in the following way.

Let $R\left(A_{1}, A_{2}, \ldots, A_{n}\right)$ be a relation scheme on domains $U_{1}, U_{2}, \ldots, U_{n}$, where $A_{i}$ is an attribute on the universe of discourse $U_{i}, i \in I$.
Denote by $\operatorname{Vag}\left(U_{i}\right)$ the set of all vague values associated to the elements $u_{i} \in U_{i}, i \in I$.

A similarity measure on $\operatorname{Vag}\left(U_{i}\right)$ is a mapping $S E_{i}: \operatorname{Vag}\left(U_{i}\right) \times \operatorname{Vag}\left(U_{i}\right) \rightarrow[0,1]$, such that $S E_{i}(x, x)=1, S E_{i}(x, y)=S E_{i}(y, x)$, and $S E_{i}(x, z) \geq \max _{y \in \operatorname{Vag}\left(U_{i}\right)}\left(\min \left(S E_{i}(x, y), S E_{i}(y, z)\right)\right)$ for all $x$, $y, z \in \operatorname{Vag}\left(U_{i}\right)$.

Suppose that $S E_{i}$ is a similarity measure on $\operatorname{Vag}\left(U_{i}\right)$, $i \in I$.

Let

$$
\begin{aligned}
A_{i} & =\left\{\left\langle u,\left[t_{A_{i}}(u), 1-f_{A_{i}}(u)\right]\right\rangle: u \in U_{i}\right\} \\
& =\left\{a_{u}^{i}: u \in U_{i}\right\}, \\
B_{i} & =\left\{\left\langle u,\left[t_{B_{i}}(u), 1-f_{B_{i}}(u)\right]\right\rangle: u \in U_{i}\right\} \\
& =\left\{b_{u}^{i}: u \in U_{i}\right\}
\end{aligned}
$$

be two vague sets in $U_{i}$.

The similarity measure $S E\left(A_{i}, B_{i}\right)$ between the vague sets $A_{i}$ and $B_{i}$ is given by

$$
\begin{aligned}
& S E\left(A_{i}, B_{i}\right) \\
= & \min \left\{\operatorname { m i n } _ { a _ { u } ^ { i } \in A _ { i } } \left\{\operatorname { m a x } _ { b _ { u } ^ { i } \in B _ { i } } \left\{S E _ { i } \left(\left[t_{A_{i}}(u), 1-f_{A_{i}}(u)\right],\right.\right.\right.\right. \\
& {\left.\left.\left.\left[t_{B_{i}}(u), 1-f_{B_{i}}(u)\right]\right)\right\}\right\}, } \\
& \min _{b_{u}^{i} \in B_{i}}\left\{\operatorname { m a x } _ { a _ { u } ^ { i } \in A _ { i } } \left\{S E _ { i } \left(\left[t_{B_{i}}(u), 1-f_{B_{i}}(u)\right],\right.\right.\right. \\
& {\left.\left.\left.\left.\left[t_{A_{i}}(u), 1-f_{A_{i}}(u)\right]\right)\right\}\right\}\right\} . }
\end{aligned}
$$

Now, if $r$ is a vague relation instance on $R\left(A_{1}, A_{2}, \ldots, A_{n}\right), t_{1}$ and $t_{2}$ are any two tuples in $r$, and $X$ is a subset of $\left\{A_{1}, A_{2}, \ldots, A_{n}\right\}$, then, the similarity measure $S E_{X}\left(t_{1}, t_{2}\right)$ between the tuples $t_{1}$ and $t_{2}$ on the attribute set $X$ has the same form as before, i.e.,

$$
S E_{X}\left(t_{1}, t_{2}\right)=\min _{A \in X}\left\{S E\left(t_{1}[A], t_{2}[A]\right)\right\} .
$$

Note that the assertions 1) and 2) remain valid if we take them with respect to new similarity measures. Namely, it is obvious that the proofs of these assertions do not depend on the choice of function $S E: V\left(U_{i}\right) \times V\left(U_{i}\right)$ $\rightarrow[0,1]$ (see, [10]).

The assertion 3), however, does not hold anymore. In particular, the following assertion holds true.

Lemma 1 Let $R\left(A_{1}, A_{2}, \ldots, A_{n}\right)$ be a relation scheme on domains $U_{1}, U_{2}, \ldots, U_{n}$, where $A_{i}$ is an attribute on the universe of discourse $U_{i}, i \in I$. Let $r$ be a vague relation instance on $R\left(A_{1}, A_{2}, \ldots, A_{n}\right)$. If $S E_{X}\left(t_{i}, t_{j}\right) \geq \theta$ and $S E_{X}\left(t_{j}, t_{k}\right) \geq \theta$, where $t_{i}, t_{j}$ and $t_{k}$ are any three tuples in $r$, and $X$ is a subset of $\left\{A_{1}, A_{2}, \ldots, A_{n}\right\}$, then $S E_{X}\left(t_{i}, t_{k}\right) \geq \theta$. 
Proof. Since

$$
\begin{aligned}
& \min _{A \in X}\left\{S E\left(t_{i}[A], t_{j}[A]\right)\right\} \\
= & S E_{X}\left(t_{i}, t_{j}\right) \geq \theta, \\
& \min _{A \in X}\left\{S E\left(t_{j}[A], t_{k}[A]\right)\right\} \\
= & S E_{X}\left(t_{j}, t_{k}\right) \geq \theta,
\end{aligned}
$$

it follows that $S E\left(t_{i}[A], t_{j}[A]\right) \geq \theta, S E\left(t_{j}[A], t_{k}[A]\right) \geq \theta$ for all $A \in X$.

Let $A \in X$. Denote by $U \in\left\{U_{1}, U_{2}, \ldots, U_{n}\right\}$ the corresponding universe of discourse, and by $S E_{U}: \operatorname{Vag}(U)$ $\times \operatorname{Vag}(U) \rightarrow[0,1]$, the corresponding similarity measure on $\operatorname{Vag}(U)$. Suppose that $|U|=N$. We shall write $U=$ $\left\{u_{1}, u_{2}, \ldots, u_{N}\right\}$. We have,

$$
\begin{aligned}
t_{i}[A] & =\left\{\left\langle u,\left[t_{t_{i}[A]}(u), 1-f_{t_{i}[A]}(u)\right]\right\rangle: u \in U\right\} \\
& =\left\{\left\langle u_{1}, a_{1}^{i}\right\rangle,\left\langle u_{2}, a_{2}^{i}\right\rangle, \ldots,\left\langle u_{N}, a_{N}^{i}\right\rangle\right\}, \\
t_{j}[A] & =\left\{\left\langle u,\left[t_{t_{j}[A]}(u), 1-f_{t_{j}[A]}(u)\right]\right\rangle: u \in U\right\} \\
& =\left\{\left\langle u_{1}, a_{1}^{j}\right\rangle,\left\langle u_{2}, a_{2}^{j}\right\rangle, \ldots,\left\langle u_{N}, a_{N}^{j}\right\rangle\right\}, \\
t_{k}[A] & =\left\{\left\langle u,\left[t_{t_{k}[A]}(u), 1-f_{t_{k}[A]}(u)\right]\right\rangle: u \in U\right\} \\
& =\left\{\left\langle u_{1}, a_{1}^{k}\right\rangle,\left\langle u_{2}, a_{2}^{k}\right\rangle, \ldots,\left\langle u_{N}, a_{N}^{k}\right\rangle\right\} .
\end{aligned}
$$

Since $S E\left(t_{i}[A], t_{j}[A]\right) \geq \theta$ and $S E\left(t_{j}[A], t_{k}[A]\right) \geq \theta$ hold true, it follows that

$$
\begin{aligned}
& \min \left\{\operatorname { m i n } \left\{\max \left\{S E_{U}\left(a_{1}^{i}, a_{1}^{j}\right), \ldots, S E_{U}\left(a_{1}^{i}, a_{N}^{j}\right)\right\},\right.\right. \\
& \max \left\{S E_{U}\left(a_{2}^{i}, a_{1}^{j}\right), \ldots, S E_{U}\left(a_{2}^{i}, a_{N}^{j}\right)\right\}, \\
& \vdots \\
& \left.\max \left\{S E_{U}\left(a_{N}^{i}, a_{1}^{j}\right), \ldots, S E_{U}\left(a_{N}^{i}, a_{N}^{j}\right)\right\}\right\}, \\
& \min \left\{\max \left\{S E_{U}\left(a_{1}^{j}, a_{1}^{i}\right), \ldots, S E_{U}\left(a_{1}^{j}, a_{N}^{i}\right)\right\},\right. \\
& \max \left\{S E_{U}\left(a_{2}^{j}, a_{1}^{i}\right), \ldots, S E_{U}\left(a_{2}^{j}, a_{N}^{i}\right)\right\}, \\
& \left.\left.\vdots \max \left\{S E_{U}\left(a_{N}^{j}, a_{1}^{i}\right), \ldots, S E_{U}\left(a_{N}^{j}, a_{N}^{i}\right)\right\}\right\}\right\} \geq \theta, \\
& \min \left\{\max \left\{S E_{U}\left(a_{1}^{k}, a_{1}^{j}\right), \ldots, S E_{U}\left(a_{1}^{k}, a_{N}^{j}\right)\right\},\right. \\
& \min \left\{\operatorname { m i n } \left\{\max \left\{S E_{U}\left(a_{1}^{j}, a_{1}^{k}\right), \ldots, S E_{U}\left(a_{1}^{j}, a_{N}^{k}\right)\right\},\right.\right. \\
& \max \left\{S E_{U}\left(a_{2}^{j}, a_{1}^{k}\right), \ldots, S E_{U}\left(a_{2}^{j}, a_{N}^{k}\right)\right\}, \\
& \vdots \\
& \left.\left.\max \left\{S E_{U}\left(a_{N}^{j}, a_{1}^{j}\right), \ldots, S E_{U}\left(a_{2}^{k}, a_{N}^{j}\right)\right\}, S E_{U}\left(a_{N}^{j}, a_{N}^{k}\right)\right\}\right\},
\end{aligned}
$$

$$
\left.\left.\max \left\{S E_{U}\left(a_{N}^{k}, a_{1}^{j}\right), \ldots, S E_{U}\left(a_{N}^{k}, a_{N}^{j}\right)\right\}\right\}\right\} \geq \theta
$$

Hence,

$$
\max \left\{S E_{U}\left(a_{p}^{i}, a_{1}^{j}\right), \ldots, S E_{U}\left(a_{p}^{i}, a_{N}^{j}\right)\right\} \geq \theta
$$

for all $p \in\{1,2, \ldots, N\}$,

$$
\max \left\{S E_{U}\left(a_{q}^{j}, a_{1}^{i}\right), \ldots, S E_{U}\left(a_{q}^{j}, a_{N}^{i}\right)\right\} \geq \theta
$$

for all $q \in\{1,2, \ldots, N\}$,

$$
\max \left\{S E_{U}\left(a_{r}^{j}, a_{1}^{k}\right), \ldots, S E_{U}\left(a_{r}^{j}, a_{N}^{k}\right)\right\} \geq \theta
$$

for all $r \in\{1,2, \ldots, N\}$,

$$
\max \left\{S E_{U}\left(a_{s}^{k}, a_{1}^{j}\right), \ldots, S E_{U}\left(a_{s}^{k}, a_{N}^{j}\right)\right\} \geq \theta
$$

for all $s \in\{1,2, \ldots, N\}$.

Our aim is to prove that $S E\left(t_{i}[A], t_{k}[A]\right) \geq \theta$. Thus, we have to prove that

$$
\max \left\{S E_{U}\left(a_{t}^{i}, a_{1}^{k}\right), \ldots, S E_{U}\left(a_{t}^{i}, a_{N}^{k}\right)\right\} \geq \theta
$$

for all $t \in\{1,2, \ldots, N\}$, and

$$
\max \left\{S E_{U}\left(a_{w}^{k}, a_{1}^{i}\right), \ldots, S E_{U}\left(a_{w}^{k}, a_{N}^{i}\right)\right\} \geq \theta
$$

for all $w \in\{1,2, \ldots, N\}$.

Let $t \in\{1,2, \ldots, N\}$ and $w \in\{1,2, \ldots, N\}$. It is enough to prove that there exists $u \in\{1,2, \ldots, N\}$ such that

$S E_{U}\left(a_{t}^{i}, a_{u}^{k}\right) \geq \theta$, and $v \in\{1,2, \ldots, N\}$ such that $S E_{U}\left(a_{w}^{k}, a_{v}^{i}\right)$ $\geq \theta$.

Put $p=t$. By (1), there exists $x \in\{1,2, \ldots, N\}$ such that $S E_{U}\left(a_{t}^{i}, a_{x}^{j}\right) \geq \theta$. Put $r=x$. By (3), there exists $y \in$ $\{1,2, \ldots, N\}$ such that $S E_{U}\left(a_{x}^{j}, a_{y}^{k}\right) \geq \theta$. We obtain,

$$
\begin{aligned}
& S E_{U}\left(a_{t}^{i}, a_{y}^{k}\right) \\
\geq & \max _{y \in \operatorname{Vag}(U)}\left(\min \left(S E_{U}\left(a_{t}^{i}, y\right), S E_{U}\left(y, a_{y}^{k}\right)\right)\right) \\
\geq & \min \left(S E_{U}\left(a_{t}^{i}, a_{x}^{j}\right), S E_{U}\left(a_{x}^{j}, a_{y}^{k}\right)\right) \\
\geq & \min (\theta, \theta)=\theta .
\end{aligned}
$$

Thus, putting $u=y$, we conclude that $S E_{U}\left(a_{t}^{i}, a_{u}^{k}\right) \geq \theta$.

Put $s=w$. By (4), there exists $e \in\{1,2, \ldots, N\}$ such that $S E_{U}\left(a_{w}^{k}, a_{e}^{j}\right) \geq \theta$. Put $q=e$. By (2), there exists $f \in$ $\{1,2, \ldots, N\}$ such that $S E_{U}\left(a_{e}^{j}, a_{f}^{i}\right) \geq \theta$. We deduce,

$$
\begin{aligned}
& S E_{U}\left(a_{w}^{k}, a_{f}^{i}\right) \\
\geq & \max _{y \in \operatorname{Vag}(U)}\left(\min \left(S E_{U}\left(a_{w}^{k}, y\right), S E_{U}\left(y, a_{f}^{i}\right)\right)\right) \\
\geq & \min \left(S E_{U}\left(a_{w}^{k}, a_{e}^{j}\right), S E_{U}\left(a_{e}^{j}, a_{f}^{i}\right)\right) \\
\geq & \min (\theta, \theta)=\theta .
\end{aligned}
$$

Hence, putting $v=f$, we obtain that $S E_{U}\left(a_{w}^{k}, a_{v}^{i}\right) \geq \theta$.

Therefore, $S E\left(t_{i}[A], t_{k}[A]\right) \geq \theta$ for all $A \in X$. Now,

$$
\begin{aligned}
& S E_{X}\left(t_{i}, t_{k}\right) \\
= & \min _{A \in X}\left\{S E\left(t_{i}[A], t_{k}[A]\right)\right\} \geq \theta,
\end{aligned}
$$

This completes the proof. 


\section{Vague multivalued dependencies}

Let $R\left(A_{1}, A_{2}, \ldots, A_{n}\right)$ be a relation scheme on domains $U_{1}$, $U_{2}, \ldots, U_{n}$, where $A_{i}$ is an attribute on the universe of discourse $U_{i}, i \in I$. Suppose that $r$ is a relation instance on $R\left(A_{1}, A_{2}, \ldots, A_{n}\right)$. Furthermore, let $X$ and $Y$ be subsets of $\left\{A_{1}, A_{2}, \ldots, A_{n}\right\}$, and $Z=\left\{A_{1}, A_{2}, \ldots, A_{n}\right\} \backslash(X \cup Y)$.

Relation instance $r$ is said to satisfy the multivalued dependency $X \rightarrow \rightarrow Y$, if for every pair of tuples $t_{1}$ and $t_{2}$ in $r, t_{1}[X]=t_{2}[X]$ implies that there exists a tuple $t_{3}$ in $r$, such that $t_{3}[X]=t_{1}[X], t_{3}[Y]=t_{1}[Y]$, and $t_{3}[Z]=t_{2}[Z]$.

Note the following facts:

Multivalued dependencies are introduced by Fagin [7], $t_{1}[X]=t_{2}[X]$ means that $t_{1}[A]=t_{2}[A]$ for all $A \in X$, $t\left[A_{i}\right] \in U_{i}$ for all $i \in I$, and all $t \in r$,

there exists the identity relation $i_{j}: U_{j} \times U_{j} \rightarrow\{0,1\}$, $j \in I$, such that $i_{j}\left(t_{k}\left[A_{j}\right], t_{l}\left[A_{j}\right]\right)=1$ if and only if $t_{k}\left[A_{j}\right]$ $=t_{l}\left[A_{j}\right]$, and $i_{j}\left(t_{k}\left[A_{j}\right], t_{l}\left[A_{j}\right]\right)=0$ if and only if $t_{k}\left[A_{j}\right] \neq$ $t_{l}\left[A_{j}\right]$, where $t_{k}, t_{l} \in r$.

If we put $\emptyset \neq t\left[A_{i}\right] \subseteq U_{i}$ for all $i \in I$, and all $t \in r$, then the relation instance $r$ becomes a fuzzy relation instance on $R\left(A_{1}, A_{2}, \ldots, A_{n}\right)$. In this setting we are able to determine how similar (or how conformant) $t_{1}[X]$ and $t_{2}[X]$ are. More precisely, we calculate the conformance $\varphi\left(X\left[t_{1}, t_{2}\right]\right)$ of the attribute set $X$ on tuples $t_{1}$ and $t_{2}$ as

$$
\varphi\left(X\left[t_{1}, t_{2}\right]\right)=\min _{A_{k} \in X}\left\{\varphi\left(A_{k}\left[t_{1}, t_{2}\right]\right)\right\},
$$

where the conformance $\varphi\left(A_{k}\left[t_{1}, t_{2}\right]\right)$ of the attribute $A_{k}$ on tuples $t_{1}$ and $t_{2}$ is given by

$$
\begin{aligned}
& \varphi\left(A_{k}\left[t_{1}, t_{2}\right]\right) \\
= & \min \left\{\min _{x \in t_{1}\left[A_{k}\right]}\left\{\max _{y \in t_{2}\left[A_{k}\right]}\left\{s_{k}(x, y)\right\}\right\},\right. \\
& \left.\min _{x \in t_{2}\left[A_{k}\right]}\left\{\max _{y \in t_{1}\left[A_{k}\right]}\left\{s_{k}(x, y)\right\}\right\}\right\} .
\end{aligned}
$$

Here, $s_{k}: U_{k} \times U_{k} \rightarrow[0,1]$ is a similarity relation on $U_{k}$, $k \in I$, i.e., $s_{k}(x, x)=1, s_{k}(x, y)=s_{k}(y, x)$, and $s_{k}(x, z) \geq$ $\max _{y \in U_{k}}\left(\min \left(s_{k}(x, y), s_{k}(y, z)\right)\right)$ for all $x, y, z \in U_{k}$.

For the similarity-based fuzzy relational database approach, see, [2]-[4].

Now, it would be natural to state that some fuzzy relation instance $r$ on $R\left(A_{1}, A_{2}, \ldots, A_{n}\right)$ satisfies the fuzzy multivalued dependency $X \rightarrow \rightarrow_{F} Y$, if for every pair of tuples $t_{1}$ and $t_{2}$ in $r$, there exists a tuple $t_{3}$ in $r$, such that

$$
\begin{aligned}
& \varphi\left(X\left[t_{3}, t_{1}\right]\right) \geq \varphi\left(X\left[t_{1}, t_{2}\right]\right), \\
& \varphi\left(Y\left[t_{3}, t_{1}\right]\right) \geq \varphi\left(X\left[t_{1}, t_{2}\right]\right), \\
& \varphi\left(Z\left[t_{3}, t_{2}\right]\right) \geq \varphi\left(X\left[t_{1}, t_{2}\right]\right) .
\end{aligned}
$$

However, it is not so hard to select both, a fuzzy relation instance $r$ on $R\left(A_{1}, A_{2}, \ldots, A_{n}\right)$, and a fuzzy multivalued dependency $X \rightarrow \rightarrow_{F} Y, X, Y \subseteq\left\{A_{1}, A_{2}, \ldots, A_{n}\right\}$, such that $r$ satisfies $X \rightarrow \rightarrow_{F} Y$ in reality, and $X \rightarrow \rightarrow_{F} Y$ makes perfectly sense by itself, but there are tuples $t_{1}$ and $t_{2}$ in $r$, such that (5) fails for every $t_{3}$ in $r$. In other words, the scenario where some of the elements $\varphi\left(X\left[t_{3}, t_{1}\right]\right), \varphi\left(Y\left[t_{3}, t_{1}\right]\right)$ and $\varphi\left(Z\left[t_{3}, t_{2}\right]\right)$ are slightly smaller than $\varphi\left(X\left[t_{1}, t_{2}\right]\right)$ for all $t_{3} \in r$, may occur. Therefore, the condition (5) is not adequate for determining if some fuzzy relation instance satisfies some fuzzy multivalued dependency. In particular, if (5) holds true, the instance $r$ satisfies $X \rightarrow \rightarrow_{F} Y$. Otherwise, $r$ may or may not satisfy $X \rightarrow \rightarrow_{F} Y$.

Note that several authors, including Tripathy-Saxena [19] and Nakata [16], have been taken attempts in order to express the fuzzy multivalued dependencies in various fuzzy relational database models.

Sozat and Yazici [17], adapted (5) in the following way.

Let $R\left(A_{1}, A_{2}, \ldots, A_{n}\right)$ be a relation scheme on domains $U_{1}, U_{2}, \ldots, U_{n}$, where $A_{i}$ is an attribute on the universe of discourse $U_{i}, i \in I$. Suppose that $r$ is a fuzzy relation instance on $R\left(A_{1}, A_{2}, \ldots, A_{n}\right)$. Furthermore, let $X$ and $Y$ be subsets of $\left\{A_{1}, A_{2}, \ldots, A_{n}\right\}, Z=\left\{A_{1}, A_{2}, \ldots, A_{n}\right\} \backslash(X \cup Y)$, and $\theta \in[0,1]$. Fuzzy relation instance $r$ is said to satisfy the fuzzy multivalued dependency $X \rightarrow \stackrel{\theta}{\rightarrow}_{F} Y$, if for every pair of tuples $t_{1}$ and $t_{2}$ in $r$, there exists a tuple $t_{3}$ in $r$, such that

$$
\begin{aligned}
& \varphi\left(X\left[t_{3}, t_{1}\right]\right) \geq \min \left(\theta, \varphi\left(X\left[t_{1}, t_{2}\right]\right)\right), \\
& \varphi\left(Y\left[t_{3}, t_{1}\right]\right) \geq \min \left(\theta, \varphi\left(X\left[t_{1}, t_{2}\right]\right)\right), \\
& \varphi\left(Z\left[t_{3}, t_{2}\right]\right) \geq \min \left(\theta, \varphi\left(X\left[t_{1}, t_{2}\right]\right)\right) .
\end{aligned}
$$

Thus, if it happens that for some $t_{1}^{\prime}$ and $t_{2}^{\prime}$ in $r$, some of the elements $\varphi\left(X\left[t_{3}, t_{1}^{\prime}\right]\right), \varphi\left(Y\left[t_{3}, t_{1}^{\prime}\right]\right)$ and $\varphi\left(Z\left[t_{3}, t_{2}^{\prime}\right]\right)$ are smaller than $\varphi\left(X\left[t_{1}^{\prime}, t_{2}^{\prime}\right]\right)$, and the elements in

$$
\left\{\varphi\left(X\left[t_{3}, t_{1}^{\prime}\right]\right), \varphi\left(Y\left[t_{3}, t_{1}^{\prime}\right]\right), \varphi\left(Z\left[t_{3}, t_{2}^{\prime}\right]\right)\right\}
$$

that are smaller than $\varphi\left(X\left[t_{1}^{\prime}, t_{2}^{\prime}\right]\right)$ are larger than $\theta$ for all $t_{3}$ $\in r$, then, the condition (6) will be fulfilled, so the instance $r$ will satisfy $X \rightarrow \stackrel{\theta}{\rightarrow}_{F} Y$ (assuming that (6) is fulfilled for $\left.\left(t_{1}, t_{2}\right) \in r \times r,\left(t_{1}, t_{2}\right) \neq\left(t_{1}^{\prime}, t_{2}^{\prime}\right)\right)$.

The value $\theta \in[0,1]$ that appears in the notation $X$ $\rightarrow \stackrel{\theta}{\rightarrow}_{F} Y$ is called the linguistic strength of the fuzzy multivalued dependency. If $\theta=1$, the fuzzy multivalued dependency $X \rightarrow \stackrel{\theta}{\rightarrow}_{F} Y$ becomes $X \rightarrow \rightarrow_{F} Y$.

Now, reasoning as in the fuzzy case, we first state that some vague relation instance $r$ on $R\left(A_{1}, A_{2}, \ldots, A_{n}\right)$ satisfies the vague multivalued dependency $X \rightarrow_{V} Y$, if for every pair of tuples $t_{1}$ and $t_{2}$ in $r$, there exists a tuple $t_{3}$ in $r$, such that

$$
\begin{aligned}
& S E_{X}\left(t_{3}, t_{1}\right) \geq S E_{X}\left(t_{1}, t_{2}\right), \\
& S E_{Y}\left(t_{3}, t_{1}\right) \geq S E_{X}\left(t_{1}, t_{2}\right), \\
& S E_{Z}\left(t_{3}, t_{2}\right) \geq S E_{X}\left(t_{1}, t_{2}\right) .
\end{aligned}
$$

Then, we adapt (7) to the following form.

Let $R\left(A_{1}, A_{2}, \ldots, A_{n}\right)$ be a relation scheme on domains $U_{1}, U_{2}, \ldots, U_{n}$, where $A_{i}$ is an attribute on the universe of discourse $U_{i}, i \in I$. Suppose that $r$ is a vague relation instance on $R\left(A_{1}, A_{2}, \ldots, A_{n}\right)$. Furthermore, let $X$ and $Y$ be subsets of $\left\{A_{1}, A_{2}, \ldots, A_{n}\right\}, Z=\left\{A_{1}, A_{2}, \ldots, A_{n}\right\} \backslash(X \cup Y)$, and $\theta \in[0,1]$. Vague relation instance $r$ is said to satisfy the vague multivalued dependency $X \rightarrow \stackrel{\theta}{\rightarrow}_{V} Y$, if for every 
pair of tuples $t_{1}$ and $t_{2}$ in $r$, there exists a tuple $t_{3}$ in $r$, such that

$$
\begin{aligned}
& S E_{X}\left(t_{3}, t_{1}\right) \geq \min \left(\theta, S E_{X}\left(t_{1}, t_{2}\right)\right), \\
& S E_{Y}\left(t_{3}, t_{1}\right) \geq \min \left(\theta, S E_{X}\left(t_{1}, t_{2}\right)\right), \\
& S E_{Z}\left(t_{3}, t_{2}\right) \geq \min \left(\theta, S E_{X}\left(t_{1}, t_{2}\right)\right) .
\end{aligned}
$$

If $\theta=1$, the vague multivalued dependency $X \rightarrow_{\rightarrow}^{\rightarrow}{ }_{V} Y$ becomes $X \rightarrow \rightarrow_{V} Y$.

For yet another definition of vague multivalued dependency, called $\alpha$-vague multivalued dependency, see [15].

Note that by [10], $r$ satisfies the vague functional dependency $X \stackrel{\theta}{\rightarrow} V Y$, if for every pair of tuples $t_{1}$ and $t_{2}$ in $r$

$$
S E_{Y}\left(t_{1}, t_{2}\right) \geq \min \left(\theta, S E_{X}\left(t_{1}, t_{2}\right)\right) .
$$

$X \stackrel{\theta}{\rightarrow}_{V} Y$ becomes $X \rightarrow_{V} Y$ if $\theta=1$.

\section{Soundness of inference rules for vague multivalued dependencies}

The following rules are the inference rules for vague multivalued dependencies (VMVDs).

VM1 Inclusive rule for VMVDs: If $X \rightarrow{\stackrel{\theta_{1}}{\rightarrow}}_{V} Y$ holds, and $\theta_{1} \geq \theta_{2}$, then $X \rightarrow{\stackrel{\theta_{2}}{\longrightarrow}}_{V} Y$ holds.

VM2 Complementation rule for VMVDs: If $X$ $\rightarrow \stackrel{\theta}{\rightarrow}_{V} Y$ holds, then $X \rightarrow \stackrel{\theta}{\rightarrow}_{V} Q$ holds, where $Q=$ $\left\{A_{1}, A_{2}, \ldots, A_{n}\right\} \backslash(X \cup Y)$.

VM3 Augmentation rule for VMVDs: If $X \rightarrow \stackrel{\theta}{\rightarrow}{ }_{V} Y$ holds, and $W \supseteq Z$, then $W \cup X \rightarrow \stackrel{\theta}{\rightarrow}_{V} Y \cup Z$ also holds.

VM4 Transitivity rule for VMVDs: If $X \rightarrow{\stackrel{\theta_{1}}{\rightarrow}}_{V} Y$ and $Y \rightarrow \stackrel{\theta_{2}}{\rightarrow} V Z$ hold true, then $X \stackrel{\min \left(\theta_{1}, \theta_{2}\right)}{\rightarrow} \rightarrow{ }_{V} Z \backslash Y$ holds true.

VM5 Replication rule: If $X \stackrel{\theta}{\rightarrow} V Y$ holds, then $X$ $\rightarrow \stackrel{\theta}{\rightarrow} V$ Y holds.

VM6 Coalescence rule for VFDs and VMVDs: If $X \rightarrow \stackrel{\theta_{1}}{\rightarrow} Y$ holds, $Z \subseteq Y$, and for some $W$ disjoint from $Y$, we have that $W{\stackrel{\theta_{2}}{\rightarrow}}_{V} Z$ holds true, then $X$ $\stackrel{\min \left(\theta_{1}, \theta_{2}\right)}{\rightarrow}{ }_{V} Z$ also holds true.

In [10], we listed the inference rules for vague functional dependencies. There, we proved that the rules are sound, and that the set of these rules, i.e., the set $\{V F 1, V F 2, V F 3, V F 4\}$ is complete set. Additional inference rules (labeled as $V F 5, V F 6$ and $V F 7$ ) are also proved to be sound.

Since the proofs of the corresponding theorems in [10] do not depend on the choice of similarity measures between vague values and vagues sets, these theorems remain valid in the present setting, i.e., for the choice: $S E_{i}$, $i \in I, S E$, and $S E_{X}$.
Theorem 2 The inference rules: VM1,VM2,VM3, VM4, VM5 and VM6 are sound.

Proof. (proof for VM1) It is enough to prove that $r$ satisfies $X \rightarrow{\stackrel{\theta_{2}}{\rightarrow}}_{V} Y$, if $r$ satisfies $X \rightarrow{\stackrel{\theta_{1}}{\rightarrow}}_{V} Y$, where $r$ is any vague relation instance on $R\left(A_{1}, A_{2}, \ldots, A_{n}\right)$.

Assume that $r$ satisfies $X \rightarrow{\stackrel{\theta_{1}}{\rightarrow}}_{V} Y$. $Y$

Furthermore, assume that $r$ does not satisfy $X \rightarrow \stackrel{\theta_{2}}{\rightarrow} V$

Since $r$ does not satisfy $X \rightarrow{\stackrel{\theta_{2}}{\rightarrow}}_{V} Y$, we know that there are tuples $t_{1}$ and $t_{2}$ in $r$, such that

$$
\begin{aligned}
& S E_{X}\left(t_{3}, t_{1}\right) \geq \min \left(\theta_{2}, S E_{X}\left(t_{1}, t_{2}\right)\right), \\
& S E_{Y}\left(t_{3}, t_{1}\right) \geq \min \left(\theta_{2}, S E_{X}\left(t_{1}, t_{2}\right)\right), \\
& S E_{Z}\left(t_{3}, t_{2}\right) \geq \min \left(\theta_{2}, S E_{X}\left(t_{1}, t_{2}\right)\right)
\end{aligned}
$$

don't hold at the same time for any $t_{3} \in r$.

Since $t_{1}$ and $t_{2}$ belong to $r$, and $r$ satisfies $X \rightarrow{\stackrel{\theta_{1}}{\rightarrow}}_{V} Y$, it follows that there exists a tuple $t_{3}$ in $r$ such that

$$
\begin{aligned}
& S E_{X}\left(t_{3}, t_{1}\right) \geq \min \left(\theta_{1}, S E_{X}\left(t_{1}, t_{2}\right)\right), \\
& S E_{Y}\left(t_{3}, t_{1}\right) \geq \min \left(\theta_{1}, S E_{X}\left(t_{1}, t_{2}\right)\right), \\
& S E_{Z}\left(t_{3}, t_{2}\right) \geq \min \left(\theta_{1}, S E_{X}\left(t_{1}, t_{2}\right)\right) .
\end{aligned}
$$

The fact that $\theta_{1} \geq \theta_{2}$ yields that

$$
\min \left(\theta_{1}, S E_{X}\left(t_{1}, t_{2}\right)\right) \geq \min \left(\theta_{2}, S E_{X}\left(t_{1}, t_{2}\right)\right) .
$$

Hence,

$$
\begin{aligned}
& S E_{X}\left(t_{3}, t_{1}\right) \geq \min \left(\theta_{2}, S E_{X}\left(t_{1}, t_{2}\right)\right), \\
& S E_{Y}\left(t_{3}, t_{1}\right) \geq \min \left(\theta_{2}, S E_{X}\left(t_{1}, t_{2}\right)\right), \\
& S E_{Z}\left(t_{3}, t_{2}\right) \geq \min \left(\theta_{2}, S E_{X}\left(t_{1}, t_{2}\right)\right) .
\end{aligned}
$$

This is a contradiction.

We conclude, $r$ satisfies $X \rightarrow{\stackrel{\theta_{2}}{\longrightarrow}}_{V} Y$.

(proof for VM3) Suppose that $r$ is a vague relation instance on $R\left(A_{1}, A_{2}, \ldots, A_{n}\right)$, such that $r$ satisfies $X \rightarrow \stackrel{\theta}{\rightarrow} V$ $Y$, and violates $W \cup X \rightarrow \stackrel{\theta}{\rightarrow} V \cup Z$.

Since $r$ violates $W \cup X \rightarrow \stackrel{\theta}{\rightarrow}_{V} Y \cup Z$, it follows that there exist tuples $t_{1}$ and $t_{2}$ in $r$, such that

$$
\begin{aligned}
S E_{W \cup X}\left(t_{3}, t_{1}\right) & \geq \min \left(\theta, S E_{W \cup X}\left(t_{1}, t_{2}\right)\right), \\
S E_{Y \cup Z}\left(t_{3}, t_{1}\right) & \geq \min \left(\theta, S E_{W \cup X}\left(t_{1}, t_{2}\right)\right), \\
S E_{\left\{A_{1}, A_{2}, \ldots, A_{n}\right\} \backslash(W \cup X \cup Y)}\left(t_{3}, t_{2}\right) & \geq \min \left(\theta, S E_{W \cup X}\left(t_{1}, t_{2}\right)\right)
\end{aligned}
$$

don't hold at the same time for any tuple $t_{3} \in r$.

Now, $t_{1}, t_{2} \in r$, and $r$ satisfies $X \stackrel{\theta}{\rightarrow}{ }_{V} Y$, hence, there is $t_{3} \in r$, such that

$$
\begin{aligned}
S E_{X}\left(t_{3}, t_{1}\right) & \geq \min \left(\theta, S E_{X}\left(t_{1}, t_{2}\right)\right), \\
S E_{Y}\left(t_{3}, t_{1}\right) & \geq \min \left(\theta, S E_{X}\left(t_{1}, t_{2}\right)\right), \\
S E_{\left\{A_{1}, A_{2}, \ldots, A_{n}\right\} \backslash(X \cup Y)}\left(t_{3}, t_{2}\right) & \geq \min \left(\theta, S E_{X}\left(t_{1}, t_{2}\right)\right) .
\end{aligned}
$$

Since $X \subseteq W \cup X$, we have that $S E_{X}\left(t_{1}, t_{2}\right) \geq$ $S E_{W \cup X}\left(t_{1}, t_{2}\right)$. Therefore,

$\min \left(\theta, S E_{X}\left(t_{1}, t_{2}\right)\right) \geq \min \left(\theta, S E_{W \cup X}\left(t_{1}, t_{2}\right)\right)$. 
Hence,

$$
\begin{aligned}
S E_{X}\left(t_{3}, t_{1}\right) & \geq \min \left(\theta, S E_{W \cup X}\left(t_{1}, t_{2}\right)\right), \\
S E_{Y}\left(t_{3}, t_{1}\right) & \geq \min \left(\theta, S E_{W \cup X}\left(t_{1}, t_{2}\right)\right), \\
S E_{\left\{A_{1}, A_{2}, \ldots, A_{n}\right\} \backslash(X \cup Y)}\left(t_{3}, t_{2}\right) & \geq \min \left(\theta, S E_{W \cup X}\left(t_{1}, t_{2}\right)\right) .
\end{aligned}
$$

The last inequality and the fact that $W \backslash(X \cup Y) \subseteq$ $\left\{A_{1}, A_{2}, \ldots, A_{n}\right\} \backslash(X \cup Y)$ yield that

$$
S E_{W \backslash(X \cup Y)}\left(t_{3}, t_{2}\right) \geq \min \left(\theta, S E_{W \cup X}\left(t_{1}, t_{2}\right)\right) .
$$

Obviously,

$$
S E_{W \cup X}\left(t_{2}, t_{1}\right) \geq \min \left(\theta, S E_{W \cup X}\left(t_{1}, t_{2}\right)\right) .
$$

Hence, $W \backslash(X \cup Y) \subseteq W \cup Y$ implies that

$$
S E_{W \backslash(X \cup Y)}\left(t_{2}, t_{1}\right) \geq \min \left(\theta, S E_{W \cup X}\left(t_{1}, t_{2}\right)\right) .
$$

Now, (8) and (9) give us

$$
S E_{W \backslash(X \cup Y)}\left(t_{3}, t_{1}\right) \geq \min \left(\theta, S E_{W \cup X}\left(t_{1}, t_{2}\right)\right) .
$$

This inequality, and the inequalities:

$$
\begin{aligned}
& S E_{X}\left(t_{3}, t_{1}\right) \geq \min \left(\theta, S E_{W \cup X}\left(t_{1}, t_{2}\right)\right), \\
& S E_{Y}\left(t_{3}, t_{1}\right) \geq \min \left(\theta, S E_{W \cup X}\left(t_{1}, t_{2}\right)\right)
\end{aligned}
$$

yield that

$$
\begin{aligned}
& S E_{W \cup X \cup Y}\left(t_{3}, t_{1}\right) \\
= & \min _{A \in W \cup X \cup Y}\left\{S E\left(t_{3}[A], t_{1}[A]\right)\right\} \\
= & \min \left(\min _{A \in W \backslash(X \cup Y)}\left\{S E\left(t_{3}[A], t_{1}[A]\right)\right\},\right. \\
& \min _{A \in X}\left\{S E\left(t_{3}[A], t_{1}[A]\right)\right\}, \\
& \left.\min _{A \in Y}\left\{S E\left(t_{3}[A], t_{1}[A]\right)\right\}\right) \\
= & \min \left(S E_{W \backslash(X \cup Y)}\left(t_{3}, t_{1}\right), S E_{X}\left(t_{3}, t_{1}\right), S E_{Y}\left(t_{3}, t_{1}\right)\right) \\
\geq & \min \left(\theta, S E_{W \cup X}\left(t_{1}, t_{2}\right)\right) .
\end{aligned}
$$

Hence, $W \cup X \subseteq W \cup X \cup Y$ gives us

$$
S E_{W \cup X}\left(t_{3}, t_{1}\right) \geq \min \left(\theta, S E_{W \cup X}\left(t_{1}, t_{2}\right)\right) .
$$

Moreover, $Y \cup Z \subseteq W \cup X \cup Y$ gives us

$$
S E_{Y \cup Z}\left(t_{3}, t_{1}\right) \geq \min \left(\theta, S E_{W \cup X}\left(t_{1}, t_{2}\right)\right) .
$$

Finally,

$$
S E_{\left\{A_{1}, A_{2}, \ldots, A_{n}\right\} \backslash(X \cup Y)}\left(t_{3}, t_{2}\right) \geq \min \left(\theta, S E_{W \cup X}\left(t_{1}, t_{2}\right)\right),
$$

and the fact that $\left\{A_{1}, A_{2}, \ldots, A_{n}\right\} \backslash(W \cup X \cup Y) \subseteq$ $\left\{A_{1}, A_{2}, \ldots, A_{n}\right\} \backslash(X \cup Y)$ imply that

$$
\begin{aligned}
& S E_{\left\{A_{1}, A_{2}, \ldots, A_{n}\right\} \backslash(W \cup X \cup Y)}\left(t_{3}, t_{2}\right) \\
\geq & \min \left(\theta, S E_{W \cup X}\left(t_{1}, t_{2}\right)\right) .
\end{aligned}
$$

Now, the inequalities (10), (11) and (12) taken together lead to contradiction.

We conclude, $r$ satisfies $W \cup X \rightarrow \stackrel{\theta}{\rightarrow}_{V} Y \cup Z$.

The cases VM2, VM4-VM6 are discussed similarly.

This completes the proof.

\section{Soundness of additional inference rules for vague multivalued dependencies}

The following inference rules are additional inference rules for vague multivalued dependencies.

VM7 Union rule for VMVDs: If $X \rightarrow{\stackrel{\theta_{1}}{\rightarrow}}_{V} Y$ and $X \rightarrow \stackrel{\theta_{2}}{\rightarrow} V Z$ hold true, then $X \stackrel{\min \left(\theta_{1}, \theta_{2}\right)}{\rightarrow} V Y \cup Z$ holds true.

VM8 Pseudo-transitivity rule for VMVDs: If $X$ $\rightarrow{\stackrel{\theta_{1}}{\rightarrow}}_{V} Y$ and $W \cup Y \rightarrow \stackrel{\theta_{2}}{\rightarrow} V$ hold true, then $W$ $\cup X \stackrel{\min \left(\theta_{1}, \theta_{2}\right)}{\rightarrow}{ }_{V} Z \backslash(W \cup Y)$ holds also true.

VM9 Decomposition rule for VMVDs: If $X \rightarrow \stackrel{\theta_{1}}{\longrightarrow} V$ $Y$ and $X \rightarrow \stackrel{\theta_{2}}{\rightarrow} V Z$ hold true, then $X \stackrel{\min \left(\theta_{1}, \theta_{2}\right)}{\rightarrow} \stackrel{十}{\longrightarrow}_{V} Y \cap Z$, $X \stackrel{\min \left(\theta_{1}, \theta_{2}\right)}{\rightarrow}{ }_{V} Y \backslash Z$, and $X \stackrel{\min \left(\theta_{1}, \theta_{2}\right)}{\rightarrow}{ }_{V} Z \backslash Y$ hold also true.

VM10 Mixed pseudo-transitivity rule: If $X \rightarrow \stackrel{\theta_{1}}{\rightarrow} V$ $Y$ and $X \cup Y \stackrel{\theta_{2}}{\rightarrow} V$ hold true, then $X \stackrel{\min \left(\theta_{1}, \theta_{2}\right)}{\rightarrow}{ }_{V} Z \backslash$ $Y$ holds true.

Theorem 3 The inference rules: VM7, VM8,VM9 and VM10 are sound.

Proof. Follows by successive application of the inference rules: VF1-VF4, and VM1-VM6.

\section{References}

[1] J. F. Baldwin, Knowledge engineering using a fuzzy relational inference language, IFAC Proceedings Volumes 16, 14-20 (1983)

[2] B. P. Buckles, F. E. Petry, A fuzzy representation of data for relational databases, Fuzzy Sets and Systems 7, 213-216 (1982)

[3] B. P. Buckles, F. E. Petry, Fuzzy databases and their applications, Fuzzy Inform. Decision Processes 2, 361-371 (1982)

[4] B. P. Buckles, F. E. Petry, Uncertainty models in information and database systems, J. Inform. Sci. 11, 77-87 (1985)

[5] S. M. Chen, Similarity Measures Between Vague Sets and Between Elements, IEEE Transactions on Systems, Man and Cybernetics 27, 153-159 (1997)

[6] S. M. Chen, Measures of Similarity Between Vague Sets, Fuzzy Sets and Systems 74, 217-223 (1995)

[7] R. Fagin, Multivalued dependencies and a new normal form for relational databases, ACM Trans. Database Systems 3, 262-278 (1977)

[8] C. Giardina, I. Sack, D. Sinha, Fuzzy Field Relational Database Tech. Report 8332 (Elect. Engng. and Computer Science Dept., Stevens Institute of Technology, Hoboken, NJ, 1983) 
[9] P. Grzegorzewski, Distances Between Intuitionistic Fuzzy Sets and/or Interval-valued Fuzzy Sets Based on the Hausdorff Metric, Fuzzy Sets and Systems 148, 319-328 (2004)

[10] Dz. Gusic, Soundness and Completeness of Inference Rules for New Vague Functional Dependencies, submitted

[11] D. H. Hong, C. Kim, A note on Similarity Measures Between Vague Sets and Between Elements, Information Sciences 115, 83-96 (1999)

[12] M. Levene, G. Loizou, A Guided Tour of Relational Databases and Beyond (Springer-Verlag, London, 1999)

[13] F. Li, Z. Xu, Measures of Similarity Between Vague Sets, Journal of Software 12, 922-927 (2001)

[14] A. Lu, W. Ng, Managing Merged Data by Vague Functional Dependencies, In: Atzeni, P., Chu, W., Lu, H., Zhou, S., Ling, T.-W. (eds.) ER 2004 LNCS, vol. 3288, pp. 259-272, Springer-Verlag, Berlin Hei- delberg, (2004)

[15] J. Mishra, S. Ghosh, A Vague Multivalued Data Dependency, Fuzzy Inf. Eng. 4, 459-473 (2013)

[16] M. Nakata, Dependencies in Fuzzy Databases: Multi-valued Dependency, In: Proceedings of IEEE Int. Conf. on Fuzzy Systems, (FUZZ-IEEE 96), pp. 1591-1597, (1996)

[17] M. Sozat, A. Yazici, A complete axiomatization for fuzzy functional and multivalued dependencies in fuzzy database relations, Fuzzy Sets and System 117, 161-181 (2001)

[18] E. Szmidt, J. Kacprzyk, Distances Between Intuitionistic Fuzzy Sets, Fuzzy Sets and Systems 114, 505-518 (2000)

[19] R. C. Tripathy, P. C. Saxena, Multivalued dependencies in fuzzy relational databases, Fuzzy Sets and Systems 58, 267-279 (1990)

[20] J. D. Ullman, Primciples of Database Systems (Computer Science Press, Rockville, MD, 1982) 